\title{
LEVANTAMENTO FLORÍSTICO DE BRYOPSIDA DE CERRADO E MATA RIPÍCOLA DO PARQUE NACIONAL DE SETE CIDADES, PIAUÍ, BRASIL ${ }^{1}$
}

\author{
Nívea Maria Carneiro Farias Castro ${ }^{2}$ \\ Kátia Cavalcanti Pôrto ${ }^{3}$ \\ Olga Yano ${ }^{4}$ \\ Antônio Alberto Jorge Farias Castro ${ }^{2}$
}

Recebido em 05/10/00. Aceito em 01/08/01.

\begin{abstract}
RESUMO - (Levantamento florístico de Bryopsida de cerrado e mata ripícola do Parque Nacional de Sete Cidades, Piauí, Brasil). Foi realizado o levantamento florístico das Bryopsida dos ecossistemas de cerrado e de mata ripícola do Parque Nacional de Sete Cidades localizado nos municípios de Piracuruca e Brasileira (04 $05-15^{\circ} \mathrm{S}$ e 4130-45'W), Piauí, Brasil. Foram identificadas 22 espécies de musgos pertencentes às famílias: Bartramiaceae (1 sp.), Bryaceae (2 sp.), Calymperaceae (2 sp.), Dicranaceae (3 sp.), Erpodiaceae (1 sp.), Fissidentaceae (6 sp.), Hypnaceae (1 esp.), Leucobryaceae (1 sp.), Pottiaceae (2 sp.), Sematophyllaceae (2 sp.) e Stereophyllaceae (1 sp.). Constituem-se novas referências para o Brasil, Weisiopsis nigeriana (Egun. \& Olar.) Zand.; para o Nordeste, Campylopus heterostachys (Hampe) Jaeg. e para o Estado do Piauí: Bryum capillare Hedw., Bryum cruegeri Hampe ex C.Müller, Philonotis uncinata (Schwaegr.) Brid., Vesicularia vesicularis (Schwaegr.) Broth., Sematophyllum subsimplex (Hedw.) Mitt., Trichosteleum fluviale (Mitt.) Jaeg., Hyophila involuta (Hook.) Jaeg. \& Sauerb., Calymperes palisotii Schwaegr. ssp. richardii (C.Müller) S.Edwards, Fissidens guianensis Mont., Fissidens intermedius C.Müller, Fissidens prionodes Mont., Fissidens goyazensis Broth. e Fissidens zollingeri Mont. Aos táxons são indicadas referências de descrições, ilustração e distribuição geográfica.
\end{abstract}

Palavras-chave - musgos, Bryopsida, cerrado, Piauí

ABSTRACT - (Bryopsida from the cerrado and gallery forest of the Sete Cidades National Park, in the State of Piauí, Brazil). This study is a floristic survey of the Bryopsida from the "cerrado" and from the gallery forest of the Sete Cidades National Park in the municipalities Piracuruca and Brasileira, in the State of Piauí, Brazil (04 $05-15^{\prime} \mathrm{S}$ and $\left.41^{\circ} 30-45^{\prime} \mathrm{W}\right)$. Twenty-two taxa were determined from the following families: Bartramiaceae (1 esp.), Bryaceae (2 esp.), Calymperaceae ( 2 esp.), Dicranaceae (3 esp.), Erpodiaceae (1 esp.), Fissidentaceae (6 esp.), Hypnaceae (1 esp.), Leucobryaceae (1 esp.), Pottiaceae (2 esp.), Sematophyllaceae (2 esp.) and Stereophyllaceae (1 esp.). Weisiopsis nigeriana (Egun. \& Olar.) Zand. is the first record of this species to Brazil,

\footnotetext{
${ }^{1}$ Parte da Dissertação de Mestrado do primeiro autor.

${ }^{2}$ Núcleo de Referência em Ciências Ambientais do Trópico Ecotonal do Nordeste (TROPEN), PRPPG/UFPI, Av.

Universitária, 1310, Campus da Ininga, CEP 64049-550, Teresina, Piauí, Brasil, e-mail_ajcastro@wpoint.com.br.

${ }^{3}$ Departamento de Botânica, CCB/UFPE, Av. Prof. Moraes Rêgo, s/n, Cidade Universitária, CEP 50670-901,

Recife, Pernambuco, Brasil.

${ }^{4}$ Instituto de Botânica, C. Postal 4005, CEP 01061-970, São Paulo, São Paulo, Brasil.
} 
Campylopus heterostachys (Hampe) Jaeg. is the first of this species found in Brazilian northeast, and Bryum capillare Hewd., Bryum cruegeri Hampe ex C.Müller, Philonotis uncinata (Schwaegr.) Brid., Vesicularia vesicularis (Schwaegr.) Broth., Sematophyllum subsimplex (Hedw.) Mitt., Trichosteleum fluviale (Mitt.) Jaeg., Hyophila involuta (Hook.) Jaeg. \& Sauerb., Calymperes palisotii Schwaegr. ssp. richardii (C.Müller) S.Edwards, Fissidens guianensis Mont., Fissidens intermedius C.Müller, Fissidens prionodes Mont., Fissidens goyazensis Broth. and Fissidens zollingeri Mont. are new to the State of Piauí. For each taxon a description is given along with references, illustrations and geographical distribution.

Keys words - mosses, Bryopsida, cerrado, Piauí

\section{Introdução}

As briófitas são plantas avasculares, pequenas e de estrutura relativamente simples. Podem ser encontradas nos habitats mais diversos, colonizando tipos variados de substratos, como muros, rochedos e areia, troncos vivos e mortos, folhas e cupinzeiros. Considerando os principais tipos de vegetação brasileira, as briófitas ocorrem tanto em matas úmidas e sombreadas como em cerrados e caatingas (Yano 1984; Lisboa 1993).

No Brasil, a maioria dos estudos sobre briófitas é de caráter florístico, que registram novas ocorrências e atualizam a distribuição geográfica dos táxons. No que se refere especificamente aos musgos, informações sobre as espécies estão compiladas nos relevantes catálogos de Yano $(1981,1989,1995)$. De acordo com os trabalhos florísticos realizados, principalmente, por Vital (1980), Marinho (1987), Pôrto (1990), Yano (1992), Lisboa (1993), Valdevino (1994), Costa (1995) e Oliveira e Silva (1998), é possível acompanhar o aumento das contribuições sobre a flora briofítica do Brasil.

Para o Nordeste, a mais recente avaliação sobre a briologia foi feita por Pôrto (1996). Este trabalho, menciona um histórico, as publicações das últimas décadas que citam coleções da região e artigos sobre distribuição geográfica de muitos táxons.

O trabalho de Egunyomi \& Vital (1984) analisa as briofloras da savana nigeriana e dos cerrados brasileiros, estabelecendo afinidades, a nível genérico, na tentativa de mostrar a natureza semelhante de ambas as vegetações. Neste artigo há citação de espécies de briófitas para os cerrados da Bahia, Maranhão e Piauí. Para o Piauí, nenhum trabalho similar a este foi realizado, até o momento.

Existem somente citações de coletas feitas por alguns botânicos em expedições científicas, estando todo o material depositado em herbários nacionais e estrangeiros (Vital 1983; Egunyomi \& Vital 1984; Frahm 1991; Reese 1993 e Ireland \& Buck 1994).

O primeiro registro de briófitas para o Piauí foi realizado por Luetzelburg (1922/23), que relaciona 11 espécies de musgos pertencentes a 5 famílias para a Bahia e o Piauí. As espécies de briófitas registradas para o Piauí pertencem às famílias Corsiniaceae, Dicranaceae, Erpodiaceae, Exormothecaceae, Funariaceae, Helicophyllaceae, Hookeriaceae, Hypnaceae, Jungermanniaceae, Leucobryaceae e Plagiotheciaceae (Yano 1981, 1984, 1989) e Calymperaceae (Reese 1993).

Dessas espécies, três são citadas para o Parque Nacional de Sete Cidades, Campylopus griseus (Hornsch.) Jaeg., Campylopus savannarum (C.Müller) Mitt. e Campylopus surinamensis C.Müller (Frahm 1991) e Syrrhopodon prolifer Schwaegr. var. acanthoneuros (C.Müller) C.Müller (Reese 1993).

Devido a escassez de estudos sobre a brioflora do Piauí, este trabalho teve por objetivos: estudar a composição da flora briofítica de uma área de preservação no centro-norte do Piauí; contribuir para o conhecimento da brioflora (musgos) dos ecossistemas de cerrado e de mata ripícola do Brasil; fornecer subsídios para estudos futuros de florística, taxonomia e ecologia e dar início à co- 
leção de briófitas do Herbário Graziela Barroso (TEPB) da Universidade Federal do Piauí (UFPI).

\section{Material e Métodos}

Área de Estudo

O Parque Nacional de Sete Cidades criado pelo Decreto Federal de $N^{\circ} 50.744$, de 8 de junho de 1961, está situado a nordeste do estado do Piauí, nos municípios de Piracuruca e Brasileira, entre as coordenadas $04^{\circ} 05^{\prime}-15^{\prime} \mathrm{S}$ e $41^{\circ} 30^{\prime}-45^{\prime} \mathrm{W}$. Possui área de 6.221,48ha, banhado por rios de médio a baixo porte, com regime hídrico irregular, apresentando intermitência. $\mathrm{O}$ relevo é suave, típico das bacias sedimentares, altitude variando entre 100 a $300 \mathrm{~m}$, com dois tipos de solos: areias quartzosas distróficas, em locais suavemente ondulados e hidromórficos, em locais planos (IBDF 1979).

Segundo Thornthwaite \& Mather (1995 apud Varejão-Silva \& Reis 1988), o clima local é Subúmido Seco com pequeno excedente hidríco no outono. A temperatura média anual é de $26,8^{\circ} \mathrm{C}$ e a precipitação anual é de $1.200 \mathrm{~mm}$. A estação chuvosa está compreendida de janeiro a maio com precipitações mensais que variam de 143 a $295 \mathrm{~mm}$. O período mais seco concentra-se entre os meses de julho a novembro com precipitações mensais variando de 7 a $22 \mathrm{~mm}$. O excedente hidríco anual é $131 \mathrm{~mm}$ e a deficiência hidríca anual de $570 \mathrm{~mm}$.

O Parque está inserido numa faixa de contato entre o cerrado e a caatinga. Considerando-se a fisionomia da vegetação, as condições climáticas e edáficas, a cobertura dominante é de cerrado, acompanhada de manchas de cerradão, campos abertos inundáveis e matas ripícolas (IBAMA 1989).

Coleta e Identificação do Material

As amostras de briófitas foram retiradas de variados tipos de substratos, como: tronco vivo, tronco em decomposição, solo, rocha e cupinzeiro. O método de coletas preferenciais (MCP) (Castro 1994) foi utilizado na amostragem. Todos os exemplares coletados foram depositados no Herbário Graziela Barroso (TEPB) da Universidade Federal do Piauí.

Para a análise e a identificação do material utilizaram-se, principalmente, os trabalhos de Bartram (1949), Reese (1961, 1983, 1993), Florschütz (1964), Syed (1973), Griffin-III (1979), Edwards (1980), Vital (1980), Crum \& Anderson (1981), Pursell (1984), Buck \& Ireland (1985), Frahm (1991), Yano (1992), Lisboa (1993), Zander (1993) e Sharp et al. (1994). O sistema de classificação adotado neste trabalho é o proposto por Vitt (1984), com modificações de Buck \& Ireland (1985) para a família Plagiotheciaceae.

É citado para cada espécie: referência, descrição, ilustração na literatura e distribuição geográfica no Brasil. Na distribuição geográfica das espécies no Brasil, os estados estão citados por suas siglas oficiais.

\section{Resultados}

No Parque Nacional de Sete Cidades foram registradas 22 espécies de Bryopsida, pertencentes a 11 famílias. Uma lista comentada dos espécies é apresentada a seguir.

\section{BRYACEAE}

Bryum apiculatum Schwaegr., Musc. Suppl. 1(2): 102. 1816.

Descrição e ilustração: Florschütz (1964); Griffin III (1979); Marinho (1987) como Bryum cruegeri Hampe ex C.Müller.

Material estudado: BRASIL. Piauí: Municípios Piracuruca e Brasileira, Parque Nacional de Sete Cidades; rocha, não fértil, N.M.Castro 12, 7-VII-1994 (TEPB 9106); rocha, não fértil, K.C.Porto s/n, 18-IV-1995 (TEPB 9263). 
Distribuição geográfica: AM, MG (Yano 1981); PB (Marinho 1987); Piauí.

Comentários: Apresenta gametófito de porte pequeno, verde-amarelado, com filídios ereto-espalhados quando secos, células hexagonais alongadas e costa percurrente a curto-excurrente. Foi encontrada em local alagado sobre rocha, associada a Bryum capillare, Campylopus savannarum e Fissidens radicans. Esta espécie está sendo citada pela primeira vez para o Piauí.

Bryum capillare Hedw., Spec. Musc. 182. 1801.

Descrição e ilustração: Syed (1973); Lisboa (1993); Sharp et al. (1994).

Material estudado: BRASIL. Piauí: Municípios Piracuruca e Brasileira, Parque Nacional de Sete Cidades; solo, não fértil, N.M.Castro 34, 7-I-1995 (TEPB 9128); cupinzeiro, não fértil, N.M.Castro 46, 9-II-1995 (TEPB 9140); rocha, não fértil, N.M.Castro 104, 12-III-1995 (TEPB 9198); rocha, não fértil, K.C.Porto s/n, 18-IV-1995 (TEPB 9219); rocha, não fértil, N.M.Castro 155,18 IV-1995 (TEPB 9249); cupinzeiro, fértil, N.M.Castro 188, 25-X-1995 (TEPB 9282).

Distribuição geográfica: RJ (Syed 1973); CE, MT, MG, SP, SC (Ochi 1980); PE, GO (Yano 1989); ES (Schäfer-Verwimp 1991); RO (Lisboa 1993); Piauí.

Comentários: A presença de rizóides no caulídio, dando uma aparência pilosa, os filídios obovado-espatulados, com borda distanta são características importantes da espécies.

Os filídios quando secos apresentam-se fortemente contorcidos sobre o caulídio. As coletas foram realizadas sobre rocha, solo arenoso e cupinzeiro, associada a Bryum apiculatum, Campylopus savannarum, C. surinamensis, Fissidens radicans, $F$. guianensis, $F$. zollingeri, Hyophila involuta, Octoblepharum albidum e Weisiopsis nigeriana. É a primeira citação para o Piauí.

\section{BARTRAMIACEAE}

Philonotis uncinata (Schwaegr.) Brid., Bryol. Univ. 2: 22. 1827.

Descrição e ilustração: Florschütz (1964); Griffin III (1979); Lisboa (1993); Sharp et al. (1994).

Material estudado: BRASIL. Piauí: Municípios Piracuruca e Brasileira, Parque Nacional de Sete Cidades; tronco vivo, não fértil, N.M. Castro 3, 7-VII-1994 (TEPB 9097); rocha, não fértil, N.M.Castro 8, 7-VII-1994 (TEPB 9102); rocha, não fértil, N.M.Castro 24, 29-X-1994 (TEPB 9118); rocha e tijolo, não fértil, col. K.C.Porto s/n, 18-IV-1995 (TEPB 9217); solo e rocha, não fértil, N.M.Castro 170, 18-IV-1995 (TEPB 9264); solo, não fértil, N.M.Castro 196, 26-X1995 (TEPB 9290).

Distribuição geográfica: AM (Griffin III 1979); MT, MG, RJ (Yano 1981); PB (Marinho 1987); PE (Pôrto 1990); ES (Schäfer-Verwimp 1991); RO (Lisboa 1993); Piauí.

Comentários: Apresenta filídios falcados, oblongo-lanceolados, com ápice acuminado, costa subpercurrente a curto-excurrente margem revoluta e serreada na metade superior (Costa 1995). Esta espécie geralmente é coletada sobre o solo ou rocha.

$\mathrm{Na}$ área estudada foi encontrada sobre tronco vivo, solo, rocha e tijolo, associada a Fissidens goyazensis e a Vesicularia vesicularis. Esta espécie está sendo citada pela primeira vez para o Piauí.

\section{STEREOPHYLLACEAE}

Entodontopsis leucostega (Brid.) Buck \& Ireland., Nova Hedwigia 41: 103. 1985.

Descrição e ilustração: Buck \& Ireland (1985); Ireland \& Buck (1994); Sharp et al. (1994).

Material estudado: BRASIL. Piauí: Municípios Piracuruca e Brasileira, Parque Nacional de Sete Cidades; tronco morto, fértil, N.M.Castro 2, 7-VII-1994 (TEPB 9096); tronco morto, não fértil, N.M.Castro 7, 7-VII-1994 
(TEPB 9101); tronco vivo, não fértil, N.M.Castro 13, 7-VII-1994 (TEPB 9107); rocha, não fértil, N.M.Castro 97, 11-II-1995 (TEPB 9191); tronco vivo, fértil, N.M.Castro 98, 12-III-1995 (TEPB 9192); tronco vivo, fértil, K.C.Porto s/n, 18-IV-1995 (TEPB 9220); tronco morto, fértil, K.C.Porto $\mathrm{s} / \mathrm{n}$, 18-IV-1995 (TEPB 9227); tronco morto, não fértil, K.C.Porto s/n, 18-IV-1995 (TEPB 9232); rocha, não fértil, K.C.Porto $\mathrm{s} / \mathrm{n}$, 18-IV-1995 (TEPB 9236); rocha, fértil, N.M.Castro 190, 25-X-1995 (TEPB 9284); tronco vivo, fértil, N.M.Castro 200, 26-X-1995 (TEPB 9294).

Distribuição geográfica: AM, BA, CE, GO, MT, MG, RJ (Yano 1981); SP (Yano \& Santos 1993); AC, DF, MA, PA, PE, PI, RO, SP (Ireland \& Buck 1994); FN (Yano 1995). Comentários: Segundo Ireland \& Buck (1994), a espécie apresenta filídios ovaladoslanceolados a oblongo-lanceolados, ápice curto acuminado e costa fraca; células alares numerosas, quadráticas a curto-retangulares, estendendo-se até próximo à costa.

Foi encontrada sobre solo, tronco vivo, tronco em decomposição, rocha, em local sombrio no interior do cerrado, em populações isoladas ou associada a Campylopus savannarum, Camlymperes palisotii subsp. richardii, Fissidens radicans, $F$. guianensis, $F$. goyazensis, Octoblepharum albidum, Sematophyllum subsimplex e Weisiopsis nigeriana. Segundo Ireland \& Buck (1994), foi citada para o município de Oeiras, Piauí, Vital 8200 (SP).

\section{HYPNACEAE}

Vesicularia vesicularis (Schwaegr.) Broth., Naturl. Pflanzenfam. 1(3): 1094. 1908.

Descrição e ilustração: Crum \& Anderson (1981); Sharp et al. (1994).

Material estudado: BRASIL. Piauí: Municípios Piracuruca e Brasileira, Parque Nacional de
Sete Cidades; rocha, não fértil, N.M.Castro 26, 8-VII-1994 (TEPB 9120).

Distribuição geográfica: PA, SC, RS (Yano 1989); SP (Visnadi \& Vital 1989); ES (Yano 1995); BA (Yano \& Bastos 1995); RJ (Oliveira e Silva 1998); Piauí.

Comentários: Espécie bastante variável, caracterizada pelos filídios largamente ovalados, curto acuminados, com células oblongo-hexagonais. Ocorre geralmente sobre solo, rocha, tronco de árvore e húmus.

No Parque foi coletada em local úmido, sobre rocha, associada a Philonotis uncinata. Esta é a primeira citação para o Piauí.

\section{SEMATOPHYLLACEAE}

Sematophyllum subsimplex (Hedw.) Mitt., J. Linn. Soc. Bot. 12: 494. 1869.

Descrição e ilustração: Griffin III (1979); Sharp et al. (1994); Buck (1998).

Material estudado: BRASIL. Piauí: Municípios Piracuruca e Brasileira, Parque Nacional de Sete Cidades, tronco morto, não fértil, N.M.Castro 5, 7-VII-1994 (TEPB 9099); rocha, não fértil, N.M.Castro 6, 7-VII-1994 (TEPB 9100); areia, não fértil, N.M.Castro 40, 7-I-1995, (TEPB 9134); tronco morto, fértil, N.M.Castro 106, 12-III-1995 (TEPB 9200); tronco vivo, fértil, N.M.Castro 110, 12-III-1995 (TEPB 9204); tronco vivo, não fértil, N.M.Castro 200, 26-X-1995 (TEPB 9294).

Distribuição geográfica: AM, PA, GO, MG, PR, RJ, RS, SC, SP (Yano 1981); PB (Marinho 1987); AP (Yano \& Lisboa 1988); MA, MT (Yano 1989); PE (Pôrto 1990); ES (Schäfer-Verwimp 1991); RR (Yano \& Mello 1992); AC (Vital \& Visnadi 1994); Piauí.

Comentários: Segundo Sharp et al. (1994), o caulídio de ramificação pinada a subpinada, conspicuamente vermelho-escuro, e filídios ereto-estendidos são características marcantes dessa espécie.

Além disso, é facilmente identificada por apresentar ramos horizontais, filídios ovalados, estreito-acuminados, células da lâmina alongadas, 
sinuosas, lisas e as alares infladas e diferenciadas em grupo de 2-4 células.

Foi coletada sobre tronco vivo, tronco em decomposição, rocha e solo arenoso, em associação com os seguintes táxons: Camlymperes palisotii subsp. richardii, Entodontopsis leucostega, Octoblepharum albidum, Syrrhopodon prolifer var. acanthoneuros. Esta é a primeira citação para o Piauí.

Trichosteleum fluviale (Mitt.) Jaeg., Ber. St. Gall. Naturw. Ges. 1876-77: 419. 1878.

Descrição e ilustração: Oliveira e Silva (1998). Material estudado: BRASIL. Piauí: Municípios Piracuruca e Brasileira, Parque Nacional de Sete Cidades; tronco vivo, fértil, N.M.Castro 16, 7-VII-1994 (TEPB 9110); rocha, não fértil, N.M.Castro 19, 7-VII-1994 (TEPB 9113); rocha, fértil, K.C.Porto s/n, 18-IV-1995 (TEPB 9216); rocha, fértil, N.M.Castro 139, 18-IV1995 (TEPB 9233).

Distribuição geográfica: AM (Griffin III 1979); MT (Yano 1981); BA (Bastos \& Yano 1993); SP (Yano \& Santos 1993); PA (Lisboa 1994); RJ (Oliveira e Silva 1998); Piauí.

Comentários: O porte reduzido, filídio largamente pontiagudo, células unipapilosas, papilas centrais não muito desenvolvidas, são características que facilitam a identificação da espécie.

Foi coletada sobre tronco vivo e, preferencialmente, sobre rocha; às vezes, associada a Campylopus savannarum, C. surinamensis, Fissidens radicans, $F$. prionodes, Octoblepharum albidum e Syrrhopodon prolifer var. acanthoneuros. Está sendo referida pela primeira vez para o Piauí.

\section{POTTIACEAE}

Hyophila involuta (Hook.) Jaeg. \& Sauerb., Ber. St. Gall. Naturw. Ges. 1871-72: 354. 1873.

Descrição e ilustração: Crum \& Anderson (1981); Zander (1993); Lisboa (1993); Sharp et al. (1994).
Material estudado: BRASIL. Piauí: Municípios Piracuruca e Brasileira, Parque Nacional de Sete Cidades; rocha, fértil, N.M.Castro 11, 7-VII-1994 (TEPB 9105); rocha, não fértil, N.M.Castro 23, 7-VII-1994 (TEPB 9117); rocha e tijolo, fértil, K.C.Pôrto, 18-IV-1995 (TEPB 9225).

Distribuição geográfica: AM, GO, PA, PR, RS, RJ (Yano 1981); PB (Marinho 1987); PE (Pôrto 1990); ES, SP (Yano 1995); Piauí.

Comentários: Filídios ovalado-lanceolados, contorcidos e com margem involuta quando secos, células apicais muito salientes na superfície superior são características desta espécie.

De modo geral, as Pottiaceae conseguem resistir à seca, ao calor e à intensa radiação através de adaptações morfológicas, como por exemplo, a presença de pêlos hialinos no ápice dos filídios e a posição dos filídios no caulídio (Zander 1993). Segundo Lisboa (1993), esta espécie é usualmente encontrada em habitat perturbado, como muros, calçadas ou paredes de concretos e tijolos.

De fato, muitos exemplares da espécie, foram coletados nos bordos das piscinas artificiais do Parque, sobre rocha, em associação com Bryum capillare, Fissidens goyazensis, F. zollingeri e Weisiopsis nigeriana. Esta é a primeira citação para o Piauí.

Weisiopsis nigeriana (Egun. \& Olar.) Zand., Bull. Buffalo. Soc. nat. Sci. 32: 193.1993. Fig. $1 \mathrm{~A}-\mathrm{H}$.

Descrição e ilustração: Egunyomi \& Olarinmoye (1978), como Gyroweisia nigeriana (Egun. \& Olar.) Zand.; Zander (1993).

Material estudado: BRASIL. Piauí: Municípios Piracuruca e Brasileira, Parque Nacional de Sete Cidades; rocha, não fértil, N.M.Castro 44, 7-I-1995 (TEPB 9138); rocha, fértil, K.C.Porto s/n, 18-IV-1995 (TEPB 9219); rocha, fértil, N.M.Castro 140, 18-IV-1995 (TEPB 9234); rocha, não fértil, K.C.Porto s/n, 18-IV-1995 (TEPB 9235); solo, fértil, N.M.Castro 160, 


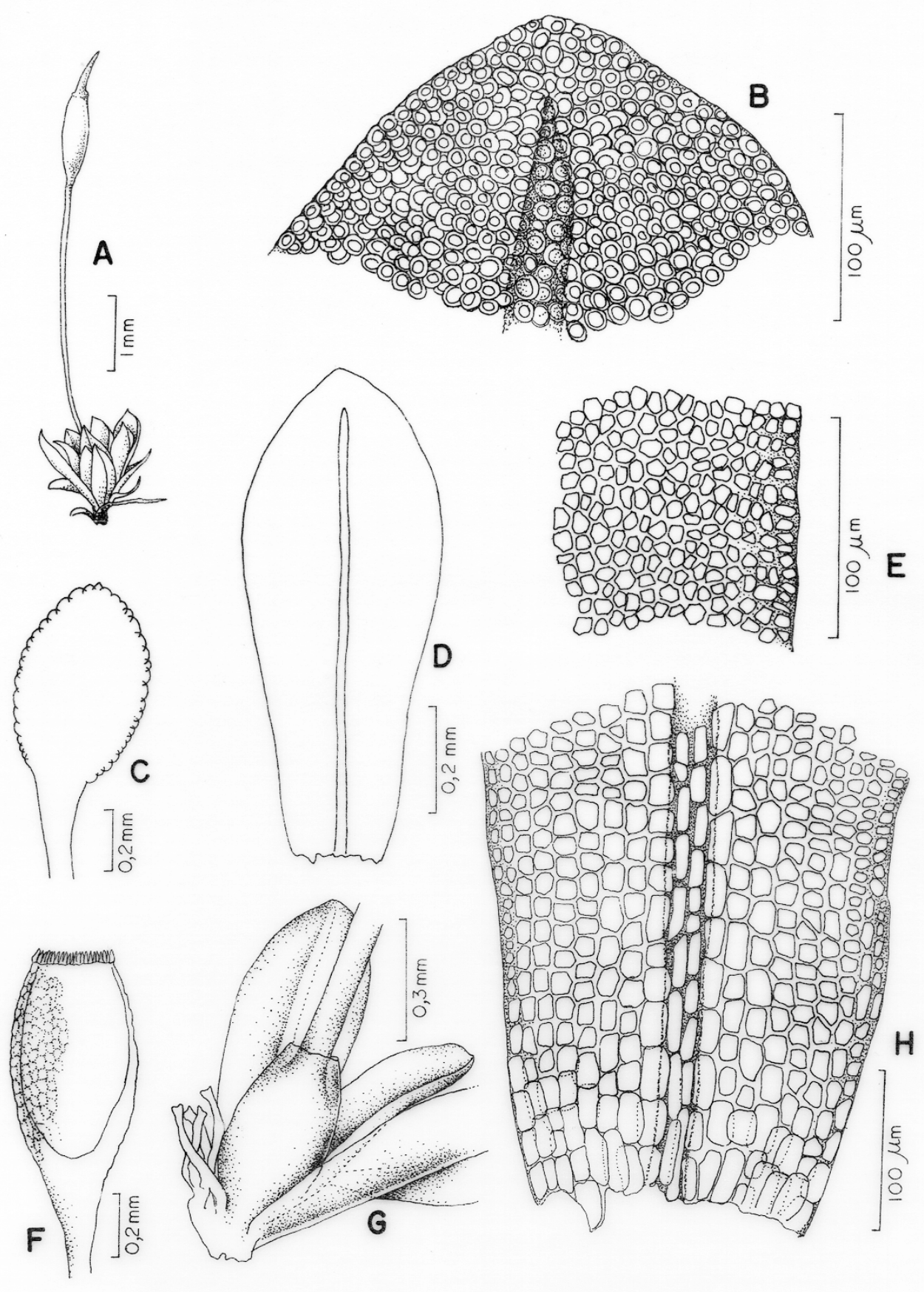

Figura 1 A-H. Weisiopsis nigeriana (Egun. \& Olar) Zand.: A. aspecto geral do gametófito; B. células apicais do filídio; C. cápsula evidenciando as células de aspecto globoso; D. filídio; E. células medianas do filídio; F. cápsula quando úmida; G. inserção da seta; H. células basais do filídio (N.M. Castro 187). 
18-IV-1995 (TEPB 9254); rocha, fértil, N.M.Castro 187, 25-X-1995 (TEPB 9281).

Comentários: A espécie foi estabelecida por Egunyomi \& Olarinmoye (1978), como Gyroweisia nigeriana, entretanto, Zander (1993) a incluiu no gênero Weisiopsis por apresentar células apicais mamilosas e dentes do peristômio espessados.

Foi coletada associada a Bryum capillare, Campylopus savannarum, $C$. surinamensis, Entodontopsis leucostega, Fissidens radicans, F. goyazensis, F. guianensis, F.zollingeri, Hyophila involuta e Octoblepharum albidum. A espécie é conhecida para a África, sendo esta a primeira citação para o Brasil.

\section{CALYMPERACEAE}

Calymperes palisotii Schwaegr. subsp. richardii (C.Müller) S.Edwards., J. Bryol. 11: 81.1980. Descrição e ilustração: Reese (1961); Florschütz (1964); Edwards(1980); Crum \& Anderson (1981); Reese (1993); Sharp et al. (1994).

Material estudado: BRASIL. Piauí: Piracuruca e Brasileira, Parque Nacional de Sete Cidades; rocha, não fértil, N.M. Castro 63, 10-II-1995 (TEPB 9157); tronco morto, não fértil, N.M.Castro 72, 10-II-1995 (TEPB 9166); tronco vivo, não fértil, N.M.Castro 86, 11-II-1995 (TEPB 9180); rocha, não fértil, N.M.Castro 128, 18-IV-1995 (TEPB 9222).

Distribuição geográfica: RO, PA, PE (Yano 1989); AP, AM, BA, FN, RN (Reese 1993); AL, ES, GO, RJ (Yano \& Costa 1993); PB, PR (Yano 1995); Piauí.

Comentários: A subespécie é identificada pelo gametófito verde-escuro de aspecto lustro, com propágulos agrupados no ápice sobre a superfície ventral do filídio; pelas cancelinas retangulares, hialinas, de paredes lisas e teníolas intramarginais, dispostas em 1-3 fileiras.

Foi coletada associada a Campylopus savannarum, Fissidens radicans, F. goyazensis, Sematophyllum subsimplex e Octoblepharum albidum. Está sendo referida pela primeira vez para o Piauí.

Syrrhopodon prolifer Schwaegr. var. acanthoneuros (C.Müller) C.Müller, Syn. Musc. frond. 1: 542.1849.

Descrição e ilustração: Florschütz (1964); Reese (1993); Lisboa (1993).

Material estudado: BRASIL. Piauí: Municípios Piracuruca e Brasileira, Parque Nacional de Sete Cidades; tronco vivo, não fértil, N.M. Castro 81, 10-I-1995 (TEPB 9175); rocha, não fértil, N.M.Castro 26, 26-X-1995 (TEPB 9285).

Distribuição geográfica: RO, PA (Yano 1989); BA, DF, ES, GO, MT, MG, PR, PI, RJ, RS, SC, SP (Reese 1993).

Comentários: Segundo Reese (1993) as variedades desta espécie são definidas pela modificação no comprimento do filídio, cor e grau de atenuação da lâmina e tamanho do gametófito. Na variedade acanthoneuros os filídios são fortemente curvados à altura dos ombros, a lâmina é usualmente verde-escura, afinando rápida $\mathrm{e}$ igualmente em direção ao ápice agudo.

Reese (1993) cita a espécie para o Piauí com base na coleta de Vital (5397, FLAS, LAF, SP) realizada no Parque Nacional de Sete Cidades. Foi coletada sobre tronco vivo e rocha, associada a Campylopus savannarum, Fissidens radicans, Octoblepharum albidum e Sematophyllum subsimplex.

\section{DICRANACEAE}

Campylopus heterostachys (Hampe) Jaeg., Ber. St. Gall. Naturw. Ges. 1870-71: 421.1872. Fig. 2 A-I.

Descrição e ilustração: Frahm (1991); Costa (1995).

Material estudado: BRASIL. Piauí: Municípios Piracuruca e Brasileira, Parque Nacional de Sete Cidades; rocha, não fértil, N.M.Castro 83, 11-II-1995 (TEPB 9177); rocha, não fértil, N.M.Castro 128, 18-IV-1995 (TEPB 9222); rocha, não fértil, N.M.Castro 155, 18-IV-1995 

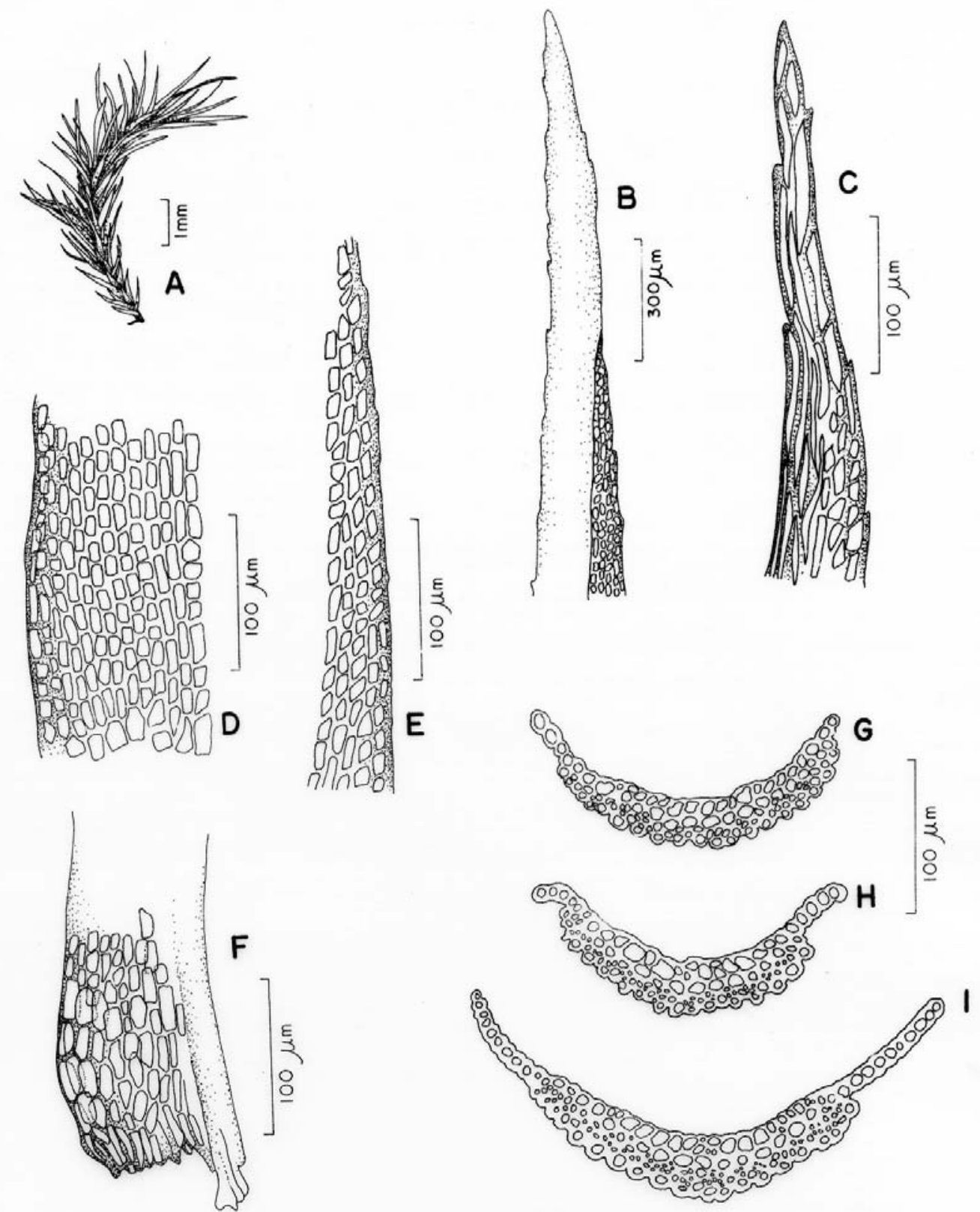

Figura 2 A-I. Campylopus heterostachys (Hampe) Jaeg.: A. aspecto geral do gametófito; B. ápice do filídio; C. detalhe do ápice evidenciando as células; D. células medianas do filídio; E. células apicais do filídio; F. células basais do filídio; G, H e I. secção transversal do filídio (N.M. Castro 155). 
(TEPB 9249); rocha, não fértil, N.M.Castro 182, 25-X-1995 (TEPB 9276).

Distribuição geográfica: SP (Yano 1981); MG (Yano 1995); RJ (Costa 1995); Piauí.

Comentários: A presença das células basais hialinas com paredes delgadas, células alares ocupando parte da costa, ápice denteado, costa em secção transversal com grandes hialocistos ventrais e grupos de pequenos estereídios dorsais são características taxonômicas que difere esta espécie de Campylopus flexuosus (Hedw.) Brid.

Ocorre sobre tronco de árvore vivas ou mortas e em paredão rochoso. Foi coletada sobre rocha, associada a Bryum capillare, $\mathrm{Ca}$ mlymperes palisotii subsp. richardii, Fissidens goyazensis e Fissidens radicans.

Está sendo citada pela primeira vez para o Nordeste do Brasil.

Campylopus savannarum (C. Müll.) Mitt., J. Linn. Soc. Bot. 12: 85.1869.

Descrição e ilustração: Florschütz (1964); Griffin III (1979); Frahm (1991); Sharp et al. (1994).

Material estudado: BRASIL. Piauí: Municípios Piracuruca e Brasileira, Parque Nacional de Sete Cidades; rocha, não fértil, N.M.Castro 10, 7-VII-1994 (TEPB 9104); rocha, não fértil, N.M.Castro 12, 7-VII-1994 (TEPB 9106); solo, não fértil, N.M.Castro 95, 11-II-1995 (TEPB 9189); rocha, não fértil, K.C.Porto s/n, 18-IV1995 (TEPB 9223).

Distribuição geográfica: AM, PE, BA (Yano 1989); PA, PI, CE, GO, MT (Frahm 1991); RJ (Costa 1995); RR (Yano 1995).

Comentários: Caracteriza-se por apresentar fileira de células quadráticas ao longo da margem basal do filídio e pela secção transversal da costa com estereídios ventrais na parte inferior do mesmo. Segundo Frahm (1991), no Brasil essa espécie é freqüente nas vegetações de cerrado e caatinga, ocorrendo também nas campinas amazônicas, que podem ser interpretadas como relito de uma savana do Pleistoceno.
O autor registra a espécie para o Piauí, coletada por Vital 5411 (SP) no Parque Nacional de Sete Cidades. Costa (1995) cita a espécie para solo arenoso, pedregoso ou semeado, rochas ou base dos troncos de árvores. Na área do Parque foi coletada sobre solo e rocha.

Campylopus surinamensis C.Müller, Linnaea 21: 186. 1848.

Descrição e ilustração: Frahm (1991).

Material estudado: BRASIL. Piauí: Municípios Piracuruca e Brasileira, Parque Nacional de Sete Cidades; rocha, não fértil, N.M.Castro 55, 10-II-1995 (TEPB 9149); rocha, não fértil, K.C.Porto s/n, 18-IV-1995 (TEPB 9235).

Distribuição geográfica: AM, BA, ES, GO, MT, MG, PA, PI, RO, SP (Frahm 1991).

Comentários: Facilmente identificada pelos filídios basais dispostos em roseta, células basais da lâmina retangulares e de paredes delgadas, tufos comais e eventualmente portar esporófitos.

Espécie comum em savanas arenosas (Frahm 1991), sendo citada para o Parque por Vital 5396 (SP). Foi coletada também sobre solo arenoso.

\section{LEUCOBRYACEAE}

Octoblepharum albidum Hedw., Spec. Musc. 50. 1801.

Descrição e ilustração: Yano (1992).

Material estudado: BRASIL. Piauí: Piracuruca e Brasileira, Parque Nacional de Sete Cidades; tronco vivo, fértil, N.M.Castro 1, 7-VII1994 (TEPB 9095); tronco morto, não fértil, N.M.Castro 27, 8-VII-1994 (TEPB 9121); tronco vivo, não fértil, N.M.Castro 28, 8-VII-1994 (TEPB 9122); raízes, não fértil, N.M.Castro 47, 9-II-1995 (TEPB 9141); rocha, não fértil, N.M.Castro 50, 9-II-1995 (TEPB 9144); areia, fértil, N.M.Castro 87, 11-II-1995 (TEPB 9181); solo, não fértil, N.M.Castro 95, 11-II-1995 (TEPB 9189); tronco vivo, fértil, K.C.Porto s/ n, 18-IV-1995 (TEPB 9220). 
Distribuição geográfica: RR, RO, AP, AC, AM, PA, MA, PI, CE, RN, PB, PE, AL, SE, BA, MT, MS, GO, MG, ES, RJ, SP, PR, SC, RS (Yano 1992).

Comentários: Caracteriza-se por apresentar filídios oblongos, com ápice denticulado, cápsula oblongo-ovalada, com 8 dentes triangulares.

Cresce em troncos de árvores vivas ou mortas, sobre pedras, solo arenoso, em lugares secos ou úmidos.

\section{FISSIDENTACEAE}

Fissidens prionodes Mont., Ann. Sci. Nat. Bot. 2(3): 200. 1835.

Descrição e ilustração: Florschütz (1964); Lisboa (1993).

Material estudado: BRASIL. Piauí: Municípios Piracuruca e Brasileira, Parque $\mathrm{Na}$ cional de Sete Cidades; rocha, não fértil, N.M.Castro 55, 10-II-1995 (TEPB 9149). Distribuição geográfica: AM, GO, MT, MG, PA, PR, RS e RJ (Yano 1981) como Fissidens hornschuchii; SC (Yano 1981), como Fissidens pellucidus; RO (Lisboa 1993); ES, FN, PE e SP (Yano 1995); Piauí. Comentários: Apresenta como principais características o porte reduzido, os filídios linear-lanceolados até elíptico-lanceolados, de ápice acuminado até apiculado, costa percurrente até curto-excurrente e células pequenas, irregulares, lisas ou finamente unipapilosas.

Segundo Lisboa (1993), esta espécie ocorre sobre solo arenoso, argiloso ou pedra, troncos podres, cupinzeiros ou até mesmo raízes de palmeiras, em diferentes tipos de vegetação como: savanas, capoeiras, matas de terra firme e florestas úmidas.

ichosteleum fluviale. Este é o primeiro registro para o Piauí.

Fissidens radicans Mont., Ann. Sci. Nat. Bot. sér. 2.14: 345. 1840.
Descrição e ilustração: Florschütz (1964); Marinho (1987); Bruggeman-Nannenga \& Pursell (1990); Sharp et al. (1994).

Material estudado: BRASIL. Piauí: Municípios Piracuruca e Brasileira, Parque Nacional de Sete Cidades; rocha, não fértil, N.M.Castro 10, 7-VII-1994 (TEPB 9104); rocha, não fértil, N.M.Castro 12, 7-VII-1994 (TEPB 9106); rocha, não fértil, N.M.Castro 83, 11-II-1995 (TEPB 9177); rocha, não fértil, N.M.Castro 84, 1-II-1995 (TEPB 9178); rocha, não fértil, K.C.Porto s/n, 18-IV-1995 (TEPB 9223); rocha, fértil, N.M.Castro 139, 18-IV-1995 (TEPB 9233); rocha, fértil, N.M.Castro 152, 18-IV-1995 (TEPB 9246); solo, não fértil, N.M.Castro 160, 18-IV-1995 (TEPB 9254); rocha, não fértil, N.M.Castro 182, 25-X-1995 (TEPB 9276).

Distribuição geográfica: PB (Marinho 1987); BA, PA, SP (Bruggeman-Nannenga \& Pursell 1990); Piauí.

Comentários: Apresenta filídios agrupados, ligeiramente contorcidos quando secos, oblongo-lingulados, ápice arredondado-obtuso, costa terminando abaixo do ápice e margem do filídio ligeiramente crenulada pelas projeções das células.

Segundo Bruggeman-Nannenga \& Pursell (1990), difere de Fissidens allenianus por apresentar filídios caducos, ápice amplamente agudo ou redondo-obtuso e peristômio reduzido. Esta espécie cresce geralmente em casca de árvores, sobre madeira morta ou raramente sobre rocha desde o nível do mar até 700m (Bruggeman-Nannenga \& Pursell 1990).

No Parque, foi coletada, sobre rocha, associada a Bryum apiculatum, B. capillare, Calymperes palisotii subsp. richardii, Entodontopsis leucostega, Fissidens elegans, F. guianensis, F. prionodes, Octoblepharum albidum, Trichosteleum fluviales, Syrrhopodon prolifer var. acanthoneuros e Weisiopsis nigeriana. Esta é a primeira citação para o Piauí. 
Fissidens zollingeri Mont., Ann. Sci. Nat. Bot., 3 (4): 144. 1845.

Descrição e ilustração: Florschütz (1964), como Fissidens kegelianus C.Müller; Marinho (1987); Lisboa (1993); Sharp et al. (1994).

Material estudado: BRASIL. Piauí: Municípios Piracuruca e Brasileira, Parque Nacional de Sete Cidades; rocha, não fértil, N.M.Castro 97, 11-II-1995 (TEPB 9191); rocha, fértil, N.M.Castro 121, 18-IV-1995 (TEPB 9215); rocha, não fértil, N.M.Castro 123, 18-IV-1995 (TEPB 9217); rocha, não fértil, N.M.Castro 159, 18-IV-1995 (TEPB 9253).

Distribuição geográfica: É citada como Fissidens kegelianus para o AM, GO, MG, PA, RJ e SC (Yano 1981); MA, PB, PE, BA, SP, RO, ES, FN, MS, PR e TO, como Fissidens zollingeri (Yano 1989, 1995); Piauí.

Comentários: Esta espécie é facilmente identificada por apresentar gametófitos reduzidos, verde-brilhantes, com filídios eqüidistantes sobre o caulídio, oblongo-lanceolados, bordeados por células longas, ápice levemente apiculado e células da lâmina vaginante bem maiores do que as células da lâmina apical.

Tem como substrato solo, madeira em decomposição e rocha calcária. Na área foi coletada somente sobre rocha. Está sendo citada pela primeira vez para o Piauí.

\section{ERPODIACEAE}

Erpodium coronatum (Hook. \& Wils.) Mitt., J. Linn. Soc. Bot. 12: 403. 1869.

Descrição e ilustração: Vital (1980); Sharp et al. (1994).

Material estudado: BRASIL. Piauí: Municípios Piracuruca e Brasileira, Parque Nacional de Sete Cidades; tronco vivo, não fértil, N.M.Castro 88, 10-II-1995 (TEPB 9182); tronco vivo, fértil, N.M.Castro 197, 26-X1995 (TEPB 9291).

Distribuição geográfica: PI (Vital 1980); BA, CE, RJ (Yano 1981); BA, PE, DF, GO, MS, MG, SP (Yano 1989); PR (Yano 1995).
Comentários: Caracteriza-se pelo gametófito verde-escuro, crescendo prostrado, filídios ovalados a largamente ovalados; quando fértil, a cápsula é ovóide-cilíndrica e imersa nos filídios periqueciais, com caliptra campanulado-mitrada e ânulo formado por 3-4 fileira de células grandes, amarelo-pálidas.

Esta espécie é citada no Piauí para Oeiras, Corrente e Cristalândia do Piauí (Vital 1980). Segundo este autor, é comumente encontrada em vegetação de cerrado e, principalmente nas áreas de transição do cerrado para outros tipos de vegetação.

Ocorre freqüentemente sobre troncos de árvores vivas, eventualmente sobre árvores em decomposição e raramente sobre rocha. No Parque Nacional de Sete Cidades foi coletada sobre tronco de Tabebuia sp.

\section{Discussão}

Os cerrados, como o do Parque Nacional de Sete Cidades, de clima subúmido e em contato com tipos de vegetação mais seca (carrasco e caatinga), apresentam uma flora briofítica significativa considerando-se o que já era conhecido para o Estado do Piauí. Dentre as espécies coletadas, $16(72,7 \%)$ referem-se a novos registros.

Das 66 famílias de musgos citadas para o Brasil, apenas nove eram assinaladas para o Piauí. Das 11 famílias registradas neste estudo, duas tinham sido citadas para o Parque Nacional de Sete Cidades e quatro para outras localidades do Estado. Bartramiaceae, Bryaceae, Fissidentaceae, Pottiaceae e Sematophyllaceae estão sendo referidas pela primeira vez para o Piauí.

Com base nos trabalhos de Luetzelburg (1922/ 23), Vital (1980), Yano (1981, 1989, 1992), Egunyomi \& Vital (1984), Frahm (1991) e Reese (1993), encontram-se registradas 14 espécies de musgos para o Piauí, das quais seis o Parque estudado. O levantamento realizado, atualiza o conhe- 
cimento da riqueza em Bryopsida, considerando que 16 espécies são acrescidas às 14 registradas anteriormente. Assim, das 30 espécies citadas para o Estado, o Parque Nacional de Sete Cidades contribui com $53,3 \%$ desse total.

Da lista de 72 espécies de musgos referidas para o cerrado brasileiro por Egunyomi \& Vital (1984): Campylopus surinamensis, C. tortilipilus, Entodontopsis leucostega (citada como Stereophyllum leucostegum), Erpodium coronatum, Fissidens zollingeri (citada também como F. kegelianus), Helicophyllum torquatum, Jonesiobryum cerradense, Octoblepharum albidum e Sematophyllum subsimplex, também ocorrem no Piauí.

Das 54 espécies relacionadas para a savana nigeriana por estes mesmos autores, apenas Erpodium coronatum, Octoblepharum albidum e Weisiopsis nigeriana (citada como Gyroweisia nigeriana), foram coletadas no cerrado do Parque Nacional de Sete Cidades. Isto significa que poucas espécies do cerrado do Piauí são comuns às de outras áreas já investigadas do cerrado brasileiro ou, mesmo da savana nigeriana, embora estes biomas apresentem condições ecológicas semelhantes (Rizzini 1997).

Para Scott (1982), em vegetações abertas onde a insolação é intensa, as briófitas são encontradas preferencialmente sobre o solo, sob casca ou tronco de árvores e arbustos, nas fendas de rochas, ou seja, em locais onde há alguma proteção contra uma rápida dessecação. No Parque as espécies, quando presentes, encontravam-se restritas a ambientes úmidos ou próximos a locais onde existia água, ocorrendo sobre base e cascas grossas dos troncos de árvores, que concentram maior umidade e onde os efeitos de escorrimento, os menores níveis de insolação e a proximidade com o solo, provavelmente garantem maior acúmulo de água.

Segundo Vitt (1981), habitats xerofíticos são colonizados por espécies de musgos tolerantes à dessecação ou por aqueles de ciclo de vida curto. Durante as coletas realizadas no Parque, apesar das populações de musgos terem sido mais conspícuas nos meses da estação chuvosa, poucas apre- sentavam esporófitos, confirmando a importância da água para a produção de esporófito.

Além disso, nos meses mais secos, com a redução do número e tamanho dos sítios ricos em umidade, observou-se a fase gametofitica dominante sobre a esporofítica.

Segundo Vitt (1979), ambientes xerofíticos e temporariamente instáveis favorecem a acrocarpia, enquanto os mesofíticos e estáveis tendem a favorecer a pleurocarpia. Esse fato também pôde ser observado no Parque, talvez por este apresentar características próximas a um ecossistema xerofítico, houve maior riqueza de espécies acrocárpicas (77\%), que de pleurocárpicas (23\%).

Com relação ao tipo de substrato, as espécies coletadas na área de estudo são preferencialmente rupícola, seguido do corticícola, terrícola e epíxila. Dos substratos analisados, a superfície rochosa apresentou maior representatividade em termos de colonização (51\%).

Isso provavelmente, deve-se a grande disponibilidade de substrato rochoso na área, com afloramentos localizados sob as sombras das árvores. Locais que retêm maior umidade propiciam um microambiente favorável ao estabelecimento das espécies como Bryum apiculatum, Campylopus heterostachys, C. surinamensis, C. savannarum, Fissidens goyazensis, F. prionodes, F. zollingeri e Vesicularia vesicularis. Na área estudada estas espécies foram coletadas apenas sobre este tipo de substrato, o que, necessariamente não indica especificidade.

De acordo com Florschütz (1964), Frahm (1991) e Lisboa (1993), estas espécies podem ocorrer em substratos variados. Para a maioria das espécies estudadas, mais de um tipo de substrato esteve associado, ainda que rocha e solo tenham sido os preferenciais.

De 299 coletas de Bryopsida realizadas, os gêneros de maior representatividade foram Fissidens $(18,7 \%)$, Octoblepharum (17\%) e Entodontopsis $(15,3 \%)$. Por sua vez, as espécies Octoblepharum albidum (17,1\%) e Entodontopsis leucostega $(15,3 \%)$, destacaram-se em representativida- 
de de amostras, seguidas de Sematophyllum subsimplex (8,4\%), Fissidens guianensis $(7,4 \%)$, Campylopus savannarum (7,0\%), Bryum capillare $(6,0 \%)$ e Weisiopsis nigeriana, Fissidens radicans e Calymperes palisotii subsp. richardii ( $6,0 \%$ cada).

\section{Agradecimentos}

Os autores agradecem o apoio logístico da administração do Parque Nacional de Sete Cidades; aos pesquisadores Dana Griffin III, R. Pursell e R.H. Zander pelas identificações e confirmações do material enviado aos seus herbários; a Dra Regina Célia Lobato do Museu Goeldi pelo apoio nas identificações e confirmações de algumas espécies; ao CNPq pela cessão de bolsa à primeira autora.

\section{Referências Bibliográficas}

Bartram, E.B. 1949. Mosses of Guatemala. Fieldiana Botany 25: 1-442.

Bastos, C.J.P. \& Yano, O. 1993. Musgos da zona urbana de Salvador, Bahia, Brasil. Hoehnea 20(1/2): 23-33.

Bruggeman - Nannenga, M.A. \& Pursell, R.A. 1990. The Fissidens radicans complex. (Section Amblyothallia) in the Neotropics and Paleotropics. Bryologist 93(3): 332-340.

Buck, W.R. 1998. Pleurocarpous Mosses of the West Indies. Memoirs of The New York Botanical Garden 1: 1-401.

Buck, W.R. \& Ireland, R.R. 1985. A reclassification of the Plagiotheciaceae. Nova Hedwigia 41: 89-125.

Castro, A.A.J.F. 1994. Comparação florísticogeográfica (Brasil) e fitossociológica (Piauí - São Paulo) de amostras de cerrado. Tese de Doutorado. Universidade Estadual de Campinas, Campinas, SP. 520p.

Costa, D.P. 1995. Musgos do município de Nova Friburgo, Rio de Janeiro, Brasil. Dissertação de Mestrado. Universidade de
São Paulo, São Paulo. 326p.

Crum, H.A. \& Anderson, L.E. 1981. Musgos of Eastern North America. Columbia, University Press, New York. 1328 p.

Edwards, S.R. 1980. A revision of west Tropical African Calymperaceae I. Introduction and Calymperes. Journal of Bryology 11: 49-93.

Egunyomi, A. \& Olarinmoye, S.O. 1978. A new Species of Gyroweisia (Musci : Pottiaceae) from Nigeria. Bryologist 81(3): 443-445.

Egunyomi, A. \& Vital, D.M. 1984. Comparative studies on the bryofloras of the Nigerian savanna and the Brazilian cerrado. Revista Brasileira Botanica 7(2): 129-36. Florschütz, P.A.1964. The mosses of Suriname. Leiden, E.J. Brill. 271 p.

Frahm, J.-P. 1991. Dicranaceae: Campylopodoideae, Paraleucobryoideae. Flora Neotropica. Monograph 54. New York, Botanical Garden. 238 p.

Griffin III, D. 1979. Guia preliminar para as briófitas freqüentes em Manaus e adjacências. Acta Amazonica 93(3): 1-67.

IBAMA. 1989. Ministério do Meio Ambiente. Unidades de conservação do Brasil; parques nacionais e reservas biológicas. v.1. Brasília: IBAMA. $182 \mathrm{p}$.

IBDF. 1979. Ministério da Agricultura. Fundação Brasileira para Conservação da Natureza. Plano de manejo do Parque Nacional de Sete Cidades. Brasília: IBDF. $61 \mathrm{p}$.

Ireland, R.R. \& Buck, W.R. 1994. Stereophyllaceae. Flora Neotropica, Monograph 65. New York, Botanical Garden. 1$49 \mathrm{p}$.

Lisboa, R.C.L. 1993. Musgos acrocárpicos do estado de Rondônia. Belém: $\mathrm{MCT} / \mathrm{CNPq} /$ Museu Paraense Emílio Goeldi. 272p. (Coleção Adolfo Ducke).

Lisboa, R.C.L. 1994. Adições à brioflora do Estado do Pará. Boletim do Museu Paraense Emílio Goeldi, sér. Bot. 10(1): 
$15-42$.

Luetzelburg, P. von. 1922/23. Estudo botânico do Nordeste. $2^{\mathrm{a}}$ ed. Rio de Janeiro. 283p.

Marinho, M.G.V. 1987. Bryopsida da reserva florestal do IBGF, João Pessoa, Paraíba, Brasil. Dissertação de Mestrado. Universidade Federal de Pernambuco. Recife, PE. 224p.

Ochi, H. 1980. A revision of the Neotropical Bryoideae, Musci (First Part). Journal Faculty of Education Tottori University 29(2): 49-154.

Oliveira e Silva, M.L.M.N. 1998. Briófitas da Reserva Ecológica de Rio das Pedras, Município de Mangaratiba, do Parque Estadual de Ilha Grande da Reserva Biológica Estadual da Praia do Sul, Município da Angra dos Reis, estado do Rio de Janeiro. Tese de Doutorado. Universidade de São Paulo. 321p.

Pôrto, K.C. 1990. Bryoflores d'une forêt de plaine et d'une forêt d'altitude moyenne dans l'état de Pernambuco (Brésil): analyse floristique. Cryptogamie, Bryologie Lichénologie 11(2): 109-161.

Pôrto, K.C. 1996. Briófitas. Pp. 97-109. In: Sampaio, E.V.S.B., Mayo, S.J. \& Barbosa, M.R.V. (Eds.) Pesquisa botânica nordestina: progresso e perspectivas. Recife, Sociedade Botânica do Brasil / Seção Regional de Pernambuco.

Pursell, R.A. 1984. A preliminary study of the Fissidens elegans complex in the Neotropics. Journal of the Hattori Botanical Laboratory 55: 235-252.

Reese, W.D. 1961. The genus Calymperes in the America. Bryologist 64(2/3): 89 -140.

Reese, W.D. 1983. American Calymperes and Syrrhopodon: Identification key and summary of recent nomenclatural changes. Bryologist 86(1): 23-30.

Reese, W.D. 1993. Calymperaceae. Flora Neotropica. Monograph 58. New York, Botanical Garden. 102p.
Rizzini, C.T. 1997. Tratado de fitogeografia do Brasil: aspectos ecológicos, sociológicos e florísticos. $2^{\mathrm{a}}$ ed. Rio de Janeiro. 747p.

Schäfer-Verwinp, A. 1991. Contribution to the knowledge of the bryophyte flora of Espírito Santo, Brazil. Journal of the Hattori Botanical Laboratory 69: 147-170.

Scott, G.A.M. 1982. Desert Bryophytes. Pp.105-122. In: Smith, A.J.E. (Ed.) Bryophyte Ecology. London: Chapman and Hall.

Sharp, A.J., Crum, H. \& Echel, P.M. 1994. The moss flora of México. Memoirs of the New York Botanical Garden 69(1-2): 1-1113.

Syed, H. 1973. A taxonomic study of Bryum capillare Hedw. and related species. Journal of Bryology 7: 265-326.

Valdevino, J.A. 1994. Bryopsida do Bituri Grande, Brejo da Madre de Deus - PE (Brasil). Dissertação de Mestrado. Universidade Federal de Pernambuco, Recife. 149p.

Varejão-Silva, M.A. \& Reis, A.C.S. 1988. Agrometeorologia e climatologia tropicais (módulo 1.1). Curso de Agricultura Tropical, O Ambiente e as Plantas Tropicais, ABEAS, Brasília.

Visnadi, S.R. \& Vital, D.M. 1989. Briófitas rupícolas de um trecho do rio Bethary, Iporanga, estado de São Paulo. Acta Botanica Brasilica 3(2): 179-83.

Vital, D.M. 1980. Erpodiaceae (Musci) do Brasil. Dissertação de Mestrado. Universidade Estadual de Campinas, Campinas, SP. 135p.

Vital, D.M. 1983. Two new species of Jonesiobryum (Musci) from the Brazilian cerrado regions. Journal of Bryology 12: 383-391.

Vital, D.M., Giancotti, C. \& Pursell, R.A. 1991. The bryoflora of Fernando de Noronha, Brasil. Tropical Bryology 4: 23-24.

Vital, D.M. \& Visnadi, S.R. 1994. Bryophytes of Rio Branco Municipality, Acre, Brazil. Tropical Bryology 9: 69-74. 
Vitt, D.H. 1979. The moss flora of the Auckland Islands, New Zealand, with a consideration of habitats, origins, and adaptations. Canadian Journal of Botany 57(20): 2226-2263.

Vitt, D.H. 1981. Adaptive modes of the moss sporophyte. Bryologist 84(2):166-186.

Vitt, D.H. 1984. Classification of the Bryopsida. Pp.696-759. In: Schuster, R.M. (Ed.) New manual of Bryology. Hattori Botanical Laboratory, Miyazaki.

Yano, O. 1981. A checklist of Brazilian mosses. Journal of the Hattori Botanical Laboratory 50:279-456.

Yano, O. 1984. Contribuição ao inventário dos Musci brasileiros: 3. Racopilaceae (Bryopsida, Isobryales). Revista brasileira Botanica 7(1/2): 57-63.

Yano, O. 1989. An additional checklist of Brazilian bryophytes. Journal of the Hattori Botanical Laboratory 66: 371-434.

Yano, O. 1992. Leucobryaceae (Bryopsida) do Brasil. Tese de Doutorado. Universidade de São Paulo, São Paulo. 318 p.

Yano, O. 1995. A new additional annotated ckecklist of Brazilian bryophytes. Journal of the Hattori Botanical Laboratory 78 : 137-182.

Yano, O. \& Lisboa, R.C. 1988. Briófitas do Território Federal do Amapá. Brasil. Boletim do Museu Paraense Emílio Goeldi, sér. bot. 4(2): 243-270.

Yano, O. \& Mello, R. 1992. Briófitas novas para o estado de Roraima, Brasil. Acta Amazonica 22(1): 23-50.

Yano, O. \& Costa, D.P. 1993. Briófitas da restinga da Massambaba, Rio de Janeiro. III Simpósio de ecossistemas da costa brasileira, Serra Negra 1992. ACIESP, V. III. São Paulo, 3:144-157.

Yano, O. \& Santos, S.X. 1993. Musgos da gruta de Mirassol, São Paulo. Acta Botanica Brasilica 7(2): 89-106.

Yano, O. \& Bastos, C.J.P. 1995. Musgos do Estado da Bahia, Brasil. Biologica Brasilica 6(1/2): 9-26.

Zander, R.H. 1993. Genera of the Pottiaceae : Mosses of Harsh Enviroments. Bulletin of the Buffalo Society of Natural Sciences 32: 171-378. 